\title{
Sprachkompetenz light?
}

\section{Christoph Hänggeli}

Rechtsanwalt, Geschäftsführer des SIWF

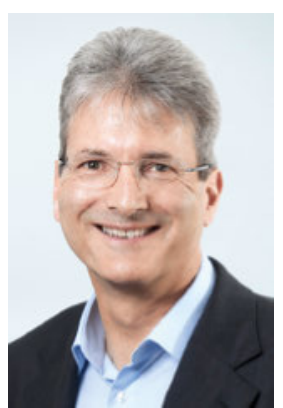

Wir erinnern uns [1]: Am 20. März 2015 haben die eidgenössischen Räte die Revision des Medizinalberufegesetzes (MedBG) definitiv verabschiedet. Dank der Unterstützung der anderen im MedBG reglementierten Berufe (Schweizerische Zahnärzte-Gesellschaft SSO, Chirosuisse, pharmaSuisse und Gesellschaft Schweizer Tierärztinnen und Tierärzte GST) ist es der FMH und dem SIWF gelungen, zwei wichtige Anliegen für den Patientenschutz zu verankern. Ab 2018 haben alle Medizinalpersonen, die in der Schweiz eine selbständige oder unselbständige Berufstätigkeit aufnehmen wollen, folgende Voraussetzungen zu erfüllen:

- Sie müssen im offiziellen Medizinalberuferegister registriert sein, womit insbesondere die amtli-

che Überprüfung ausländischer Diplome sichergestellt ist.

- Sie müssen die für die jeweilige Berufsausübung notwendigen Sprachkenntnisse nachweisen.

Die Konkretisierung und Umsetzung der minimal geforderten Sprachkenntnisse liegt im Zuständigkeitsbereich des Bundesrates. Das Bundesamt für Gesundheit (BAG) hat zu diesem Zweck eine Ausführungsverordnung ausgearbeitet und ein breites Anhörungsverfahren durchgeführt. Eine griffige Regelung zur Sicherstellung der Sprachkompetenz ist nicht in Sicht. Zwar will das Bundesamt alle nachgewiesenen Sprachkenntnisse im Register eintragen, z.B. Englisch für einen aus den USA und Kroatisch für einen aus Kroatien stammenden Arzt. Der Verordnungsentwurf verzichtet aber

Vor der Aufnahme einer Tätigkeit in der Schweiz müssen alle ausländischen Ärzte in Zukunft ihr Diplom anerkennen oder überprüfen lassen.

darauf, für unselbständig erwerbstätige Ärztinnen und Ärzte das Beherrschen einer Schweizer Landessprache zu fordern. Aus Sorge um die Arzt-Patienten-Kommunikation haben wir deshalb in der Vernehmlassung vorgeschlagen, als Minimum die Kenntnisse einer Schweizer Amtssprache auf dem Niveau B2 in der Medizinalberufeverordnung aufzunehmen; B2 basiert auf dem Gemeinsamen Europäischen Referenzrahmen und entspricht in etwa dem Maturitätsniveau.
Diese Forderung liegt immer noch weit unter den Standards, welche andere europäische Länder voraussetzen. Deutschland und Österreich beispielsweise führen strenge Sprachtests durch, in denen u.a. mit einem simulierten Patientengespräch geprüft wird, ob die medizinische Fachsprache verstanden und artikuliert werden kann. Durchfallquoten von 30\% sind an der Tagesordnung. In diesen Ländern herrscht Einigkeit darüber, dass sich Ärztinnen und Ärzte mühelos mit Patienten, aber auch Kollegen in einer Landessprache verständigen können müssen, damit Fehldiagnosen und falsche Therapieentscheidungen infolge mangelndem Sprachverständnis ausgeschlossen sind.

In anderen europäischen Ländern kann der Arztberuf ohne Beherrschung einer Landessprache nicht ausgeübt werden.

Nicht so in der Schweiz: Hier wird mit Argumenten gefochten wie «Man soll den Markt spielen lassen» oder «Die Spitäler können doch frisch engagierte Ärzte in Intensivsprachkurse schicken». In einer sensiblen Branche wie dem Gesundheitswesen können fehlende Sprachkenntnisse erwiesenermassen schnell lebensbedrohliche Folgen annehmen. Und nicht nur das: Soll die Schweiz mitten in Europa eine Insel bilden und als Zufluchtsort dienen mit Sogwirkung auf alle Ärztinnen und Ärzte, denen in den umliegenden Ländern wegen ungenügenden Sprachkenntnissen die Berufsausübung verwehrt ist?

Der Bundesrat wird voraussichtlich noch in diesem Monat über den definitiven Wortlaut der Medizinalberufeverordnung entscheiden. Wird er den Vorschlag FMH/SIWF "mindestens Niveau B2 in einer Amtssprache» aufnehmen und damit einen wesentlichen Beitrag für die Patientensicherheit und Versorgungsqualität leisten? Oder soll die Schweiz in Europa das Schlusslicht mit den tiefsten Sprachanforderungen bleiben?

\footnotetext{
Literatur

1 Schlup J, Hänggeli C. Revision des Medizinalberufegesetzes: Ende gut, alles gut? Schweiz Ärztezeitung. 2015;96(19):677.
} 